\title{
The Influences of the Quality of Sleep of the Chronic Stroke Patients on the Cognitive Function, the Motivation for the Rehabilitation, and the Capability to Carry Out the Everyday Life
}

Wongi Choi ${ }^{1}$, Juhyung Park²*

1 Department of Occupational Therapy, Rehabilitation Medicine, Cheong Ju Medical Center, 48, Heungdeok ro, Seowon gu, Cheongju si, Chungcheongbuk do, Republic of Korea 2* Department of Occupational Therapy, Health Science College, Cheongju University

\begin{abstract}
[Purpose] This research had intended to find out about the influences of the quality of sleep of the chronic stroke patients on the cognitive function, the motivation for the rehabilitation, and the capability to carry out the everyday life and their correlations.

[Subjects and Methods] This research had the 31 stroke patients who had been hospitalized or had been receiving the outpatient treatment at hospitals that are located in Korea as the subjects. To measure the quality of sleep, cognitive function, capability to carry out the activities of daily living and motivation for the rehabilitation of the subjects had been measured by using the Pittsburgh Sleep Quality Index (PSQI), the Mini Mental State Examination (MMSE-K), the Korean version of the Modified Barthel Index (K-MBI) and the rehabilitation motivation measurement tool.

[Results Although, as a result of the analysis, regarding the capability to carry out the everyday life activities and the cognitive function according to the quality of sleep, the significant differences appeared $(p<.05, p<.01)$, the motivation for the rehabilitation did not show any significant difference. It appeared that, between the quality of sleep, capability to carry out the everyday life activities, cognitive function, and motivation for the rehabilitation of the subjects, it appeared that there had been the significant correlations $(p<0.05)$.

[Conclusion] Through the results of this research, it was able to confirm that the quality of sleep of the chronic stroke patients can have the significant influences on their capability to carry out the everyday life activities, cognitive function, and motivation for the rehabilitation.

Keywords: Activities of daily living, quality of sleep, stroke
\end{abstract}

\section{Introduction}

Regarding the stroke, as an illness of the central nervous system that causes the functional disorder because the blood does not get smoothly supplied to the brain tissues because of the ischemia and the bleeding of the brain blood vessels, it has

Correspondence: juhyungi79@hanmail.net, +82-10-7751-7238 298 Daesung-ro, Cheongwon-gu, Cheongju 363-764, Republic of Korea Received: 12 April 2019 Accepted: 10 August 2019

\section{Sleep and Hypnosis}

Journal homepage:

http://www.sleepandhypnosis.org

ISSN:1302-1192 (Print) 2458-9101 (Online) been known that the disorder that takes place after the occurrence of the stroke generally appears in the complex forms in relation to the exercise and cognition functions, including the hemiplegia, the sensory disorder, the language disorder, the memory disorder, etc. (Kim, 2015; Prange, Jannink, Groothuis-udshoorn, Hermans, \& IJzerman, 2006; Zwecker et al., 2002). It has been known that, generally, regarding such stroke patients, due to the problems that were mentioned earlier, among them, the everyday life activities of $25.0 \%$ 74.0\% become reliant. (Miller, et al., 2010). Such reliant, everyday life activities can cause a lot of the problems. Kim, Kang, Kim, Wang, and Chang (2006) reported that, in case the everyday life activities which cannot be 
independent continue, because the helplessness and the depression symptom can come easily and because the reliance gets fortified, due to the result of the worsening of the interpersonal relationships, ultimately, the quality of life is lowered. Also, in case, after the stroke, the minimal, everyday activities cannot be carried out independently, as the assistance by the family members is needed and the burden of the family members can be aggravated, it is said that the improvement of the capability to carry out the everyday life activities of the chronic stroke patients possesses the very important meaning (Bang, 2007). As a result, the promotion of the capability to carry out the everyday life activities after a stroke can be a very important element in the rehabilitation of the stroke patient. And it is only through an accurate evaluation and by accurately understanding what elements have been giving the influences on the capability to carry out the everyday life activities before the medical treatment that an appropriate medication can take place by adjusting to the problem of the patient.

Generally, it has been known that the cognitive function and the motivation for the rehabilitation of the chronic stroke patients are the factors that have the big influences on their overall rehabilitations. And, regarding the cognitive function, among them, as a very important factor with regard to the medical rehabilitation treatment of the stroke patients, by having the influences on the desire for, and the motivation behind, the participation in a rehabilitation program and the capability to acquire the exercise function when the cognitive function has been damaged, it has been known to be a factor that can have a negative influence with regard to the capability to carry out the everyday life of the subjects. (Ebrahim, Nouri \& Barer, 1985). Also, regarding the motivation for the rehabilitation on the part of the stroke patients, it can be said that it invigorates the capability to survive according to the relationships with the sensory perception, the autonomy, and the environment regarding their capabilities, and it can be said to be a psychological desire for participating in an activity for quickly adapting to the changed form of the life. (Hafen, Jastrebow, Nubling, \& Bengel, 2001). The motivation for the rehabilitation has a function that makes one reach the goal after beginning a certain work and strengthening the behavior. And, when aggressively forming, because the will to receive the medical treatment on the part of the patient and the attitude of intending to aggressively engage regarding the medical treatment appear, it has the important action on the process or the result of the medical treatment. (Hafen, Jastrebow, Nubling, \& Bengel, 2001).

Regarding the sleep, as a mechanism for recovering the functions of the body and for maintaining the strength and the health, it is recharged physically and emotionally. And it is the most important method for the rest in the condition of the unconsciousness from which one can wake up because of the appropriate sensation or stimulation (Foreman \& Wykle, 1995). Also, it has been known that the normal sleep structure plays an important role, especially, in not only the learning and the memory, but, also, in the acquisition of the exercise techniques. And this means that the sleep is important with regard to the recovery of a stroke patient. (Djonlagic, Saboisky, Carusona, Stickgold, \& Malhotra, 2012).

However, after the occurrence of stroke, most of the patients show the sleep problem, and it is said that, when comparing with the people to whom the stroke did not occur, the quality of the sleep is markedly worse (Campos, et al,. 2013). Such a sleep disorder after a stroke has the influences on the function during the daytime, the quality of life, and the processes of the neurological illnesses like the death rate (Sterr, Herron, Dijk \& Ellis, 2008). Also, for the participation in a rehabilitation program, a lot of the energy is needed. Sleep is a natural and preferred method for obtaining the energy (Bakken, Kim, Arnstein, \& Anners, 2012).

However, regarding the stroke patients, because the quality of sleep gets reduced, the drowsiness during the daytime increases, and they complain of fatigue. And, due to these, the result of obtaining the negative influence on the overall rehabilitation can be caused. (Gottselig, Bassetti, \& Achermann, 2002). Despite the fact that such a sleep disorder is a problem that takes place frequently to the stroke patients, the scientific and clinical interests are on the side of being small. And, after the sleep disorder 
being manifested as a temporary symptom, there are the many cases in which it is naturally recovered. And it is thought that, rather than being a problem due to the illness, there had been a lot of the cases which are regarded as the emotional elements, including the anxiety, the depression, etc. (Kim, 2015).

As such, in this research, it had been intended to find out about the influences of the quality of sleep of the stroke patients on the cognitive function, the motivation for the rehabilitation, and the capability to carry out the everyday life of the subjects. And it had been intended to find out about the correlations between each variables that can have the influences on the rehabilitation of the patients.

\section{METHODS}

\section{Participants and Procedure}

This research had the stroke patients who had been hospitalized or had been receiving the outpatient treatments at the 5 hospitals that are located in Korea. And the selection criteria had been the following: The persons who received the diagnosis as a stroke in the Magnetic Resonance Imaging (MRI) or the Computerized Tomography (CT); The persons whose communication is possible at 24 points or higher in the MMSE-K; The persons who do not have any illnesses other than the stroke; The persons who have not been participating in a different research; And the persons who understood the purpose of this research and consented to the participation. The number of the subjects were calculated by using the G-power version 3.1. And, as a result of calculating by setting up 0.3 for the effect size, 0.8 for the power, and 0.5 for the significance level, because the final 27 persons were required, during the proceeding with the program for the 4 weeks, by considering the withdrawals, the 52 persons had been recruited. Among these, the 21 subjects who did not conform to the selection criteria were excluded. And, in the final research, a total of the 31 persons had participated. This research had been proceeded with after acquiring the approval (1041107-201812-HR-03402) of the Deliberation Committee of the Research Ethics Committee of the Cheongju University.

When collecting the materials, after the inspector personally explained the purpose and the object of the research and after obtaining the written consent to the participation in the research, the subjects who were suitable to the selection criteria had been made to respond by using a written survey questionnaire. By using the Electronic Medical Records (EMR's), the special, general characteristics of the age, the gender, the time period of the illness, the type of the stroke, and the hemisphere of the lesion had been investigated. And the MBI, MMSE-K, PSQI, and rehabilitation motivation investigations had been carried out. Regarding the inspections, the 5 occupational therapists of each organization had carried them out. And, regarding the written survey questionnaires, they were personally entered, or, regarding the subjects who had a difficulty in entering personally, the inspector personally read the written survey questionnaire and made them respond. Thereby, with a total of the 31 persons as the subjects, the written survey questionnaires had been written.

\section{INSTRUMENTS}

\section{Pittsburgh Sleep Quality Index(PSQI)}

In order to evaluate the quality of the sleep, the PSQI had been used. As a self-reporting tool, the Pittsburgh Sleep Quality Index was organized with the 19 questions. And each item was distinguished into the 7 organizational elements as the following: The subjective quality of the sleep (1 question); The time it takes to go to sleep (2 questions); The time of the continuation of the sleep (1 question); The efficiency of the everyday life sleep (3 questions); The problems related to sleep (9 questions); The use of the drugs related to sleep (1 question); And the dysfunctions during the daytime (2 questions). And each question was measured at o point $\sim 3$ points. And, the total score was o point 21 points. In case it is 5 points or higher, it means that the quality of the sleep is bad. (Buyse, Reynold, Monk, Berman \& Kupfer, 1989). It was reported that, at the time of the development, regarding the level of the confidence, the Cronbach a value was 0.83 .

\section{Korean version of the Mini Mental State Examination (MMSE-K)}

It is the evaluation tool after Kwon and Park 
(1989) revised and supplemented the Mini-Mental State Examination (MMSE) that was developed by Folstein (1975). And it is organized with the 6 items, including the orientation, the memory registration, the memory recollection, the caution concentration and calculation, the language function, and the understanding and the judgment. With the 12 questions, the lowest is o point and the highest is 30 points. And, regarding the uneducated elderly, while correcting, too, by adding 1 point to the orientation regarding the time, 2 points to the caution concentration and calculation, and 1 point to the language function, there had been the corrections so that the full score is not surpassed regarding each question. It is classified that 24 points or higher means the normal, cognitive function, 18 points-23 points means the mild dementia, and 17 points or lower means the moderate and the severe dementia. At the time of the development, regarding the level of the confidence of the tool, the Cronbach's value was 0.86 .

\section{Korean version of the Modified Barthel Index (K-MBI)}

In order to evaluate the capability carry out the everyday life activities, the K-MBI had been used. The thing that resulted after Shah, Vanclay, and Cooper (1989) revised and supplemented the Barthel Index (BI) that was developed for evaluating the level of the everyday self-reliance of the chronically ill in order to measure the extents of the everyday life activities of the stroke patients is the Modified Barthel Index (MBI). In this research, the K-MBI, which Jung et al. (2007) developed by revising and translating the MBI Version 5 to fit the actual circumstances of Korea, had been used. Regarding the evaluation items, they were the following 10 items: Dining, personal hygiene, bathing, toilet disposal, climbing up the staircase, wearing the clothes, the adjustment of the urine and the feces, walking or chair car, and the movement of the chair and the bed. From the minimum of o point to the maximum of 100 points, the closer to 100 points, one is more reliant on the everyday life activities. Regarding the levels of the reliabilities between the inspectors of the Korean writing version, revised, Barthel Index
0.93 0.98 was reported, and the Cronbach a value of 0.84 was reported (Jung et al., 2007).

\section{Rehabilitation Motivation Measurement Tool}

For the tool for measuring the motivation for the rehabilitation, the tool that was developed by Han and Lim (2002) in order to measure the motivation for the rehabilitation on the part of the disabled had been used. This tool was organized with a total of the 27 questions, including the 8 questions on the task-oriented motivation, the 7 questions on the change-oriented motivation, the 4 questions on the obligatory motivation, the 4 questions on the external motivation, and the 4 questions on the amotivation. Regarding each question, on the 5-point, Likert scale, 'Very much so' is 5 points, and 'Not at all so' is 1 point. With 135 points being the highest, the higher the score is, it means that the motivation for the rehabilitation is high. At the time of the development, regarding the level of the reliability of the tool, the Cronbach's a value was o.86.

\section{DATA ANALYSIS}

For the statistical analyses of this research, the SPSS Version 18.0 program had been used. Regarding the special, general characteristics and the special, clinical characteristics of the subjects, the frequency analysis had been used. And, regarding the differences between the capability to carry out the everyday life activities, the cognitive function, and the rehabilitation motivation resulting from the quality of the sleep, the independent sample t-test had been used. In order to find out the correlations between the variables, the Pearson Correlation Coefficient had been carried out. The statistically significant level $\alpha$ was set up at 0.05 .

\section{RESULTS}

The results of the analysis of the special, general characteristics of the subjects in this research are as the following (Table 1): Regarding the gender, there had been a lot of the females a 17 persons (54.8\%), and, regarding the gender, those who were aged between 50 years old and 59 years old had shown the 
Table 1. General Characteristics of Participants

\begin{tabular}{|c|c|c|c|c|c|}
\hline \multicolumn{2}{|c|}{ Characteristics } & \multirow{2}{*}{$\begin{array}{c}\text { Subjects(n) } \\
14\end{array}$} & \multirow{2}{*}{$\begin{array}{c}\text { Percentage(\%) } \\
45.2\end{array}$} & \multirow{3}{*}{$\begin{array}{c}\text { Average } \\
-\end{array}$} & \multirow{3}{*}{$\begin{array}{c}\text { Standard deviation } \\
-\end{array}$} \\
\hline Condor & Male & & & & \\
\hline Genaer & Female & 17 & 54.8 & & \\
\hline \multirow{6}{*}{ Age } & $30 \sim 39$ & 2 & 6.5 & \multirow{6}{*}{61.81} & \multirow{6}{*}{13.49} \\
\hline & $40 \sim 49$ & 4 & 12.9 & & \\
\hline & $50 \sim 59$ & 9 & 29.0 & & \\
\hline & $60 \sim 69$ & 7 & 22.6 & & \\
\hline & $70 \sim 79$ & 7 & 22.6 & & \\
\hline & $80 \sim 89$ & 2 & 6.5 & & \\
\hline \multirow{2}{*}{ Stroke type } & Infarction & 21 & 67.7 & \multirow{2}{*}{-} & \multirow{2}{*}{ - } \\
\hline & Hemorrhage & 10 & 32.3 & & \\
\hline \multirow{3}{*}{$\begin{array}{c}\text { Stroke } \\
\text { hemisphere }\end{array}$} & Right & 13 & 41.9 & \multirow{3}{*}{-} & \multirow{3}{*}{-} \\
\hline & Left & 16 & 51.6 & & \\
\hline & Both & 2 & 6.5 & & \\
\hline \multirow{4}{*}{$\begin{array}{c}\text { On set } \\
(\mathrm{mo})\end{array}$} & $6 \sim 11$ & 17 & 54.8 & \multirow{4}{*}{13.23} & \multirow{4}{*}{5.82} \\
\hline & $12 \sim 17$ & 7 & 22.6 & & \\
\hline & $18 \sim 23$ & 6 & 19.4 & & \\
\hline & $24 \sim$ & 1 & 3.2 & & \\
\hline
\end{tabular}

Table 2. The Difference of MBI, MMSE-K,

Rehabilitation Motivation according to PSQI

PSQI

\begin{tabular}{ccc} 
& $\begin{array}{c}\text { Good sleep quality } \\
\mathrm{N}=14 / \mathrm{M} \pm \mathrm{SD}\end{array}$ & $\begin{array}{c}\text { Bad sleep quality } \\
\mathrm{N}=17 / \mathrm{M} \pm \mathrm{SD}\end{array}$ \\
\hline $\mathrm{MBI}^{* *}$ & $71.21 \pm 5.01$ & $56.00 \pm 17.65$ \\
\hline MMSE-K* $^{*}$ & $26.86 \pm 1.70$ & $23.71 \pm 2.78$ \\
\hline $\begin{array}{c}\text { Rehabilitation } \\
\text { Motivation }\end{array}$ & $84.86 \pm 5.30$ & $80.59 \pm 6.73$ \\
\hline
\end{tabular}

${ }^{*} \mathrm{p}<.05,{ }^{* *} \mathrm{p}<.01$

PSQI: Pittsburgh Sleep Quality Index, MBI:

Modified Barthel Index, MMSE-K: Korean

version of Mini-Mental State Examination 
Table 3. The correlation among ADL ability, PSQI, Cognitive, Motivation $(n=31)$

\begin{tabular}{|c|c|c|c|c|c|}
\hline & & PSQI & MBI & MMSE-K & $\begin{array}{c}\text { Rehabilitation } \\
\text { Motivation }\end{array}$ \\
\hline \multirow[t]{3}{*}{ PSQI } & $r$ & 1 & & & \\
\hline & $p$ & & & & \\
\hline & $n$ & 31 & & & \\
\hline \multirow[t]{3}{*}{ MBI } & $r$ & $-.414^{\star}$ & 1 & & \\
\hline & $\mathrm{p}$ & .021 & & & \\
\hline & $n$ & 31 & 31 & & \\
\hline \multirow[t]{3}{*}{ MMSE-K } & $r$ & $-.586^{\star \star}$ & $.437^{\star}$ & 1 & \\
\hline & $\mathrm{p}$ & .001 & .014 & & \\
\hline & $n$ & 31 & 31 & 31 & \\
\hline \multirow{3}{*}{$\begin{array}{l}\text { Rehabilitation } \\
\text { Motivation }\end{array}$} & $r$ & $-365^{*}$ & $0.476^{\star \star}$ & $.455^{\star}$ & 1 \\
\hline & $\mathrm{p}$ & .044 & .007 & .010 & \\
\hline & $\mathrm{n}$ & 31 & 31 & 31 & 31 \\
\hline
\end{tabular}

${ }^{*} \mathrm{p}<.05,{ }^{* *} \mathrm{p}<.01$

MBI : Modified Barthel Index, PSQI : Pittsburgh Sleep Quality Index, MMSE-K : Korean version of Mini-Mental State Examination

highest distribution at 9 persons (29.0\%). Regarding the type of the stroke, it appeared as the 21 persons (67.7\%) for the bleeding and the 10 persons (32.3\%) for the ischemic. And, regarding the hemisphere of the damage of the stroke, it appeared as 13 persons (41.9\%) for the right side, 16 persons (51.6\%) for the left side, and 2 persons (6.5\%) for the both sides. Regarding the time periods of the occurrence of the illness, they were 6 months $\sim 11$ months for the 17 persons (54.8\%), 12 months 17 months for the 7 persons (22.6\%), 18 months 23 months for the 6 persons (19.4\%), and 24 months or longer for the 1 person (3.2\%) (Table 1).

As a result of having compared the capability to carry out the everyday life activities, the cognitive function, and the motivation for the rehabilitation of the subjects according to the quality of the sleep, regarding the aspect of the capability to carry out the everyday life, the group that showed the high quality of the sleep showed the capability to carry out the everyday life on a significantly high level compared to the group that showed a low quality of the sleep $(\mathrm{p}<0.01)$. With regard to the cognitive function aspect, in the same way as the capability to carry out the everyday life, the group of which the quality of the sleep was high showed the significant- ly high cognitive function ( $\mathrm{p}<0.05$ ), with regard to the rehabilitation motivation aspect, no significant difference could be observed. (Table 2).

As a result of finding out the correlations between the quality of the sleep, the capability to carry out the everyday life activities, the cognitive function, and the rehabilitation motivation of the subjects, it appeared that all of the quality of the sleep, the capability to carry out the everyday life activities, the cognitive function, and the rehabilitation motivation showed the significant, negative correlations ( $p<0.01, p<0.05)$. And, regarding the correlations between the capability to carry out the everyday life activities, the cognitive function, and the time period of the re-activity, all of them showed the significant, positive correlations ( $\mathrm{p}<0.05$ ) (Table 3 ).

\section{DISCUSSION}

Although the sleep of a chronic stroke patient is a very important factor that has the influence on the results of the rehabilitation, the actual circumstance is that the researches regarding this are insufficient. As such, this research had been carried out in order to find out the influences of the quality of the sleep that has the influence on the rehabilitation of 
a stroke patient on the cognitive function, the rehabilitation motivation, and the capability to carry out the everyday life and in order to find out the importance of the sleep when rehabilitation training of the chronic stroke patients by investigating the correlations between each of the variables.

In this research, by dividing the subjects into the group of which the quality of the sleep is high and the group of which the quality of the sleep is low and their capabilities to carry out the everyday life activities had been compared. As the result of an experiment, it appeared that the group of which the quality of the sleep is high showed a significantly high capability to carry out the everyday life $(\mathrm{p}<0.01)$. And, in the result of the analysis of the correlation, too, the significant, negative correlation could be confirmed. This means that the quality of the sleep of the subjects has a correlation with the everyday life activities and is a factor that has the influence. This represents that, the worse the quality of the sleep, the more reliant the everyday life activities are. Regarding this result, the high level of the reliance of the carrying out of the everyday life that was obtained in the research by Bakken et al. (2012), which had investigated the relationships between the independence of the everyday life activities, the sleep, and the other indispensable elements, has the relationships with the short sleep time at night and a lot of the sleep time during the day. And it is similar to the result that the high number of the times of waking up during the sleep gives a negative influence to the low capability to carry out the everyday life.

In this research, in order to find out what influences the quality of the sleep of the chronic stroke patients has on the cognitive function and the rehabilitation motivation, too, which are the important factors during the rehabilitation training of the subjects, in the same way as the everyday life activities, the levels of each of the variables according to the quality of the sleep had been compared. Although, as a result of the experiment, with regard to the cognitive function aspect, in the same way as the capability to carry out the everyday life, the group of which the quality of the sleep was high showed the significantly high cognitive function $(\mathrm{p}<0.05)$, with regard to the rehabilitation motivation aspect, no significant difference could be observed. However, in the result of analyzing the correlation, regarding both of the two variables, it was able to confirm the significant, negative correlation with the quality of the sleep of the chronic stroke patients ( $\mathrm{p}<0.05)$. Although such a result is a result (Jang et al,. 2013; Oh \& Chun, 2000; Sung, Lee, Song \& Jun, 2011) that coincides with the argument in the results of the precedent researches that, regarding the aspect of the cognitive function of the chronic stroke patients, if the quality of the sleep is low, it gives the negative influences to the physiological, physical, and cognitive functions and the recovery of the functions, it can be seen to be a result that is different from the research results that had mentioned that, with regard to the rehabilitation motivation aspect, the elements like the lowering of the concentration ability, the lowering of the memory capability, the depression, the fatigue, and the lowering of the functional recovery due to a sleep disorder make one even more reliant in the everyday life activities. (Kim, Kim, Yang, Kim \& Kim, 2015; Vance, Heaton, Eaves \& Fazeli, 2011). Regarding the group of the subjects who had participated in this research, as the stroke patients who entered into the chronicity, due to the special characteristics of the illness, they could not experience a lot of the changes regarding the things that had been endeavored in the beginning. And it is thought that a significant difference of the rehabilitation motivation according to the quality of the sleep of most of the subjects was not shown because the possibility of the rehabilitation and the feeling of the self-efficacy had been lowered a lot during the long time period of the rehabilitation. But, because a significant, negative correlation was observed in the result of analyzing the correlation, it can be seen to be a result that partially supports the argument of the precedent researches.

If we take a look at the results of this research, they suggest that the quality of the sleep is a very important element that must not be overlooked during the rehabilitation training of the stroke patients. Such results had explained the significant correlation between the quality of the sleep, the cognitive function, the capability to carry out the every- 
day life activities, and the rehabilitation motivation of the stroke patients. And, because the quality of the life can have a lot of the influences on the capability to carry out the everyday life activities, the rehabilitation motivation, and the cognitive function of the chronic stroke patients, it has been suggesting that, during the rehabilitation training, the management of the quality of the sleep of the chronic stroke patients is definitely necessary. Hence, it is thought that, when the chronic stroke patients are receiving the rehabilitation training, in order to understand the quality of their sleep, the training must carried out while having the many-sided and specific guidelines for the evaluation and the management regarding the quality of the sleep.

Regarding the limitation in this research, it had been limited to the stroke patients who had been rehabilitating in the institutes in some of the regions.
And, because the number of the subjects was comparatively small, there had been the insufficiency with regard to the aspect of the generalization of the research results. Also, because the correlation between the capability to carry out the everyday life activities and the detailed domains of the cognitive function could not be found out, there had been the point of not being able to materialize the detailed analysis. As a result, in the follow-up researches, by having this research as the basic material, by recruiting more subjects from the diverse regions and organizations, the generalization of the research is needed. And it is thought that the more detailed analyses and researches regarding the detailed domains of the capability to carry out the everyday life activities, the cognitive function, and the rehabilitation motivation regarding the quality of the sleep of the stroke patients will be needed.

\section{Conflict of interest: The authors do not declare any conflict of interest.}

\section{References}

Bakken, N. L., Kim, S. H., Arnstein F., \& Anners, L. (2012). Stroke patients' functions in personal activities of daily living in relation to sleep and socio-demographic and clinical variables in the acute phase after first-time stroke and at six months of follow-up. Journal of Clinical Nursing, 21, 1886-1895. doi:10.111/ j.1365-2702.2011.04014.x.

Bang, Y. S. (2007). The effects of task-oriented activities on the cognitive function and performance of activities of daily living in stroke patients. Journal of Korean Society of Occupational Therapy, 15(3), 49-61.

Buyse, D. J., Reynold, C. F., Monk, T. H., Berman, S. R., \& Kupfer, D. J. (1989). The Ptttsburgh sleep quality index: A new instrument for psychiatric practice and research. Psychiatry Research, 28, 193-213.

Campos, T. F., Barroso, M. T. M., Silveria, A. B. G., De Melo, L. P., Dantas, A. A. T. S. G., \& Araujo, J. F. (2013). Sleep disturbances complaints in stroke: Implications for sleep medicine. Sleep Science, 6, 98-102.

Djonlagic, I., Saboisky, J., Carusona, A., Stickgold, R., \& Malhotra, A. (2012). Increased sleep fragmentation leads to impaired off-line consolidation of motor memories in humans. Public Library of Science ONE. 7, e34106. doi:10.1371/journal.pone.0034106

Ebrahim S, Nouri F, Barer D. Cognitive impairment after stroke. Age Ageing. 1985;14:345-348.

Folstein, M. F., Folstein, S. E., \& McHugh, P. R. (1975). "Mini-mental state": A practical method for grading the cognitive state of patients for the clinician. Journal of Psychiatric Research, 12(3), 189-198.
Foreman, M. D., \& Wykle, M. (1995). Nursing standard of practice protocol : sleep disturbances in elderly patients. Geriatric Nursing, 16, 238-243. doi:10.1016/So197-4572(05)80173-9

Gottselig, J. M., Bassetti, C. L., \& Achermann, P. (2002). Power and coherence of sleep spindle frequency activity following hemispheric stroke. Brain, 125, 373-383. doi:10.1093/brain/awfo21

Hafen, K., Jastrebow, J., Nubling, R., \& Bengel, J. (2001). Development of patient questionnaire for assessment of motivation for rehabilitation (PAREMO). Rehabilitation(Stuttg), 40, 3-11. doi:10.1055/s-2001-12136

Han, H. S., \& Lim, N. Y. (2002). On the test of the reliability and validity of the disabled's motivation scale for rehabilitation. Korean Journal of Rehabilitation Nursing, 5(2), 124-133.

Jang, Y. S., Kim, H. D., Chung, H. A. (2013). Correlations among the sleep, fatigue and quality of life in patients with stroke. Journal of the Korea Academia-Industrial Cooperation Society, 14, 6302-6308. doi:10.5762/KAIS.2013.14.12.6302

Jung, H. Y., Park, B. K., Shin, H. S., Kang, Y. K., Pyun, S. B., Paik, N. J., ,, Han, T. R. (2007). Development of the Korean version of Modified Barthel Index(K-MBI): Multi-center study for subjects with stroke. Journal of Korean Academy of Rehabilitation Medicine ,31, 283-297.

Kim, D. Y. (2015). Sleep disorders after stroke. Brain and NeuroRehabilitation, 8, 73-80. doi:10.12786/bn.2015.8.2.73

Kim, J. H., Kang, H. S., Kim, W. O., Wang, M. J., \& Chang, C. M. (2006). Factors affecting the quality of life in stroke patient at home. Korean Journal of Rehabilitation Nursing, 9(1), 49-55.

Kim, J. N., Kim, Y. T., Yang, K, I., Kim, D, E., \& Kim, S. A. (2015). The 
relationship between sleep disturbance and functional status in mild stroke patients. Annals of Rehabilitation Medicine, 39, 545552. doi:10.5535/arm.2015.39.4.545

Miller, E. L., Murray, C. L., Richards, L., Zorowitz, R. D., Bakas, T., Clark, P., \& Billinger, S. A. (2010). Comprehensive overview of nursing and interdisciplinary rehabilitation care of the stroke patient: scientific statement from the American heart association. Stroke, 41, 2402-2448. doi:10.1161/STR.obo13e3181e7512b

Oh, S. Y., \& Chun, M. H. (2000). Insomnia in patients during rehabilitation treatmen. Journal of Korean Academy of Rehabilitation Medicine, 24, 395-401.

Prange, G. B., Jannink, M. J. A., Groothuis-Oudshoorn, C. G. M., Hermans, H. J., \& IJzerman, M. J. (2006). Systematic review of the effect of robot-aided therapy on recovery of the hemiparetic arm after stroke. Journal of Rehabilitation Research and Development, 43, 171-184. doi:10.1682/JRRD.2005.04. 0076

Shah, S., Vanclay, F., \& Cooper, B. (1989). Improving the sensitivity of the Barthel index for stroke rehabilitation. Journal of Clinical Epidemiology, 42, 703-709.
Sterr, A., Herron, K., Dijk, D. J., \& Ellis, J. (2008). Time to wake-up: Sleep problems and daytime sleepness in long-term stroke survivors. Brain Injury, 22, 575-579. doi:10.1080/02699050802189727

Sung, M. H., Lee, M. H., Song, G. S., \& Jun, E. M. (2011). The effects of foot reflexology massage on the central pain, fatigue and sleep in stroke patients. Journal of Korean Clinical Nursing Research, 17(1), 46-56.

Vance, D. E., Heaton, K., Eaves, Y., \& Fazeli, P. L. (2011). Sleep and cognition on everyday functioning in older adults: Implications for nursing practice and research. Journal of Neuroscience Nursing, 43, 261-271. doi:10.1097/JNN.obo13e318227efb2

Zwecker, M., Levenkrohn, S., Fleisig, Y., Zeilig, G., Ohry, A., \& Adunsky, A. (2002). Mini-mental state examination, cognitive FIM instrument, and the Loewenstein occupational therapy cognitive assessment: Relation to functional outcome of stroke patients. Archives of Physical Medicine and Rehabilitation, 83, 342-345. doi:10.1053/apmr.2002.29641 